\title{
Association between adiposity indices and cardiometabolic risk factors among adults living in Puerto Rico
}

\author{
Cristina Palacios ${ }^{1, *}$, Cynthia M Pérez ${ }^{2}$, Manuel Guzmán ${ }^{3}$, Ana P Ortiz ${ }^{2,4}$, Alelí Ayala ${ }^{2}$ \\ and Erick Suárez ${ }^{2}$ \\ ${ }^{1}$ Nutrition Program, Department of Human Development, Graduate School of Public Health, Medical Sciences \\ Campus, University of Puerto Rico, PO Box 365067, San Juan, PR 00936-5067: ${ }^{2}$ Department of Biostatistics \\ and Epidemiology, Graduate School of Public Health, Medical Sciences Campus, University of Puerto Rico, \\ San Juan, Puerto Rico: ${ }^{3}$ School of Medicine, Medical Sciences Campus, University of Puerto Rico, San Juan, \\ Puerto Rico: ${ }^{4}$ Cancer Control and Population Sciences Program, University of Puerto Rico Comprehensive \\ Cancer Center, San Juan, Puerto Rico
}

Submitted 29 September 2010: Accepted 9 March 2011: First published online 24 May 2011

\begin{abstract}
Objective: To compare the general adiposity index (BMI) with abdominal obesity indices (waist circumference (WC), waist-to-hip ratio (WHR) and waist-to-height ratio (WHtR)) in order to examine the best predictor of cardiometabolic risk factors among Hispanics living in Puerto Rico.

Design: Secondary analysis of measurements taken from a representative sample of adults. Logistic regression models (prevalence odds ratios (POR)), partial Pearson's correlations (controlling for age and sex) and receiver-operating characteristic (ROC) curves were calculated between indices of obesity (BMI, WC, WHR and WHtR) and blood pressure, HDL cholesterol (HDL-C), LDL cholesterol (LDL-C), total cholesterol (TC):HDL-C, TAG, fasting blood glucose, glycosylated $\mathrm{Hb}$, high-sensitivity C-reactive protein (hs-CRP), fibrinogen, plasminogen activator inhibitor-1 (PAI-1) and an aggregated measure of cardiometabolic risk.

Setting: Household study conducted between 2005 and 2007 in the San Juan Metropolitan Area in Puerto Rico.

Subjects: A representative sample of 858 non-institutionalized adults.

Results: All four obesity indices significantly correlated with the cardiometabolic risk factors. WHtR had the highest POR for high TC:HDL-C, blood pressure, hs-CRP, fibrinogen and PAI-1; WC had the highest POR for low HDL-C and high LDL-C and fasting blood glucose; WHR had the highest POR for overall cardiometabolic risk, TAG and glycosylated $\mathrm{Hb}$. BMI had the lowest POR for most risk factors and smallest ROC curve for overall cardiometabolic risk.

Conclusions: The findings of the study suggest that general adiposity and abdominal adiposity are both associated with cardiometabolic risk in this population, although WC, WHR and WHtR appear to be slightly better predictors than BMI.
\end{abstract}

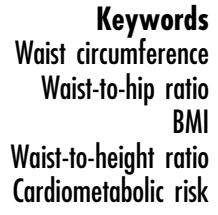

Excess adipose tissue is clearly associated with cardiometabolic risk factors, namely hypertension, dyslipidaemia and diabetes. BMI has been the obesity index most often used by clinicians and in epidemiological studies to identify individuals and populations at risk of CVD and several other chronic conditions ${ }^{(1)}$. Although computed tomography and MRI are considered the gold standard measurements for abdominal fat ${ }^{(2,3)}$, and dual-energy $\mathrm{X}$-ray absorptiometry is considered a very reliable alternative $^{(3,4)}$, these are not feasible to use in large epidemiological studies; therefore, BMI and other indices have been used. BMI has the limitation of being a measure of excess weight relative to height, rather than to excess body fat, reflecting both fat mass and lean mass.
In addition, BMI does not provide the fat distribution, which may be a more important determinant of all-cause mortality ${ }^{(5)}$. Body fat measured by abdominal fat, as measured by the indices of body fat centralization (waist circumference (WC), waist-to-hip-ratio (WHR) and waistto-height-ratio (WHtR)), appears to be more related to cardiometabolic risk factors compared with BMI. This has been shown in several studies where WC and WHR appear to be better indicators of cardiometabolic risk factors compared with BMI in Caucasians, African Americans and Asians ${ }^{(6-9)}$. However, others show no differences among obesity indices in predicting risk ${ }^{(10-12)}$. Since WC and WHR do not take into account body size, WHtR has been proposed more recently to be a better 
predictor of cardiometabolic diseases ${ }^{(13-19)}$, although there are conflicting findings ${ }^{(9,11,20-22)}$. WHtR has been proposed as a more sensitive index than BMI and WC because it is more closely associated with central obesity and it encompasses the adjustment to different statures ${ }^{(23)}$; therefore, for a given height, there is an acceptable degree of fat stored in the upper body.

The controversy regarding which obesity index is the strongest predictor of cardiometabolic risk factors is important in the clinical setting and in public health to easily and uniformly identify those at risk. In addition, the best obesity index could vary within population groups $^{(24)}$. Most studies have been conducted on Caucasians and Asians, and there are only a few studies on the Hispanic population ${ }^{(25-27)}$. Therefore, in the present study we sought to compare the general adiposity index (BMI) with abdominal obesity indices (WC, WHR and WHtR) to examine the best correlate and predictor of cardiometabolic risk factors among Puerto Rican adults.

\section{Methods}

The present study is a secondary analysis of data collected from the study 'Prevalence of the Metabolic Syndrome in San Juan, Puerto Rico', conducted from 2005 to 2007 in Puerto Rico ${ }^{(28)}$.

\section{Study population}

The study population consisted of 858 non-institutionalized Puerto Ricans aged 21-79 years residing in the San Juan Metropolitan Area, a geographical area that includes seven municipalities (population $=955431$ ) of Puerto Rico. Details of the sampling have been published recently ${ }^{(28,29)}$. In brief, the sampling frame was based on the maps of the San Juan Metropolitan Area Census tracts, and the sampling procedure was a cluster design for household surveys using a three-stage sample design. All individuals aged 21-79 years from each selected household were eligible to participate in the study and were asked to undergo a personal interview and physical examination and to have their biochemical measurements taken. Pregnant women were excluded from the study. The response rate was $72 \cdot 3 \%$.

\section{Data collection}

In the main study, measurements were taken in a mobile examination centre located near the participants' homes. These measurements included anthropometric measurements, blood pressure and fasting blood samples.

\section{Anthropometric measurements}

Anthropometric measurements were taken in duplicate according to the National Health and Nutrition Examination Survey III Anthropometric Video Procedures, and the average of the two measures was used (Centers for Disease Control and Prevention, Atlanta, GA, USA). Body weight was measured in kilograms using a digital scale
(Cardinal, Detecto, Webb City, MO, USA) and height was measured in metres using a portable stadiometer (Seca Corporation, Hanover, MD, USA). WC was determined using a measuring tape at the high point of the iliac crest at minimal respiration in centimetres, and the following categories were used: high risk $\geq 102 \mathrm{~cm}$ in men and $\geq 88 \mathrm{~cm}$ in women and low risk if below these levels ${ }^{(30)}$. Hip circumference (HC) was determined using a measuring tape at the maximum extension of the buttocks at minimal respiration in centimetres. BMI was calculated as weight in kilograms divided by the square of height in metres $\left(\mathrm{kg} / \mathrm{m}^{2}\right)$, and the following categories were used: underweight $\left(<18.5 \mathrm{~kg} / \mathrm{m}^{2}\right)$, normal weight $\left(18.5-24.9 \mathrm{~kg} / \mathrm{m}^{2}\right)$, overweight $\left(25 \cdot 0-29 \cdot 9 \mathrm{~kg} / \mathrm{m}^{2}\right)$ and obese $\left(\geq 30 \cdot 0 \mathrm{~kg} / \mathrm{m}^{2}\right)^{(31)}$. WHR was calculated as WC $(\mathrm{cm})$ divided by $\mathrm{HC}(\mathrm{cm})$, and the following categories were used: high risk $\geq 0.90$ in men and $\geq 0.85$ in women and low risk if below these levels ${ }^{(32)}$. WHtR was calculated as WC $(\mathrm{cm})$ divided by height $(\mathrm{cm})$, and the following categories were used: high risk $\geq 0.5$ in men and women and low risk if below these levels ${ }^{(33)}$.

\section{Blood pressure}

Three blood pressure measurements were taken $10 \mathrm{~min}$ apart using an appropriate cuff size and a standard aneroid sphygmomanometer. Before the measurement, participants were asked to sit quietly in a chair for at least 5 min, with feet on the floor and arms supported at chest level. Blood pressure status was based on the average of the three measurements. High blood pressure was defined as systolic blood pressure (SBP) $\geq 130 \mathrm{mmHg}$ and diastolic blood pressure (DBP) $\geq 85 \mathrm{mmHg}^{(34)}$.

\section{Fasting blood samples}

After the participants had fasted for $10 \mathrm{~h}$, blood samples were obtained from an antecubital vein while participants were sitting. Blood samples were collected, centrifuged, refrigerated at the mobile examination centre and transferred in iceboxes to a local reference laboratory on the day of the analyses. Concentrations of total cholesterol (TC), TAG, HDL cholesterol (HDL-C), fasting plasma glucose and glycosylated $\mathrm{Hb}$ were determined using commercial enzymatic colorimetric kits (Bayer Diagnostics, Tarrytown, NY, USA). Levels of LDL cholesterol (LDL-C) were estimated indirectly using the Friedewald equation. A two-site immunoassay for measuring human fibrinogen in plasma was used (DiaPharma Group Inc., West Chester, OH, USA). Plasminogen activator inhibitor-1 (PAI-1) levels were determined using the Imubind enzyme-linked immunosorbent assay (American Diagnostica Inc., Stamford, CT, USA). High-sensitivity CRP (hs-CRP) was measured using the ultrasensitive assay (Kamiya Biomedical, Seattle, WA, USA). On the basis of national guidelines, low HDL-C was defined as levels $\leq 40 \mathrm{mg} / \mathrm{dl}$ in men and $\leq 50 \mathrm{mg} / \mathrm{dl}$ in women $^{(35)}$; high TC:HDL-C was defined as levels $\geq 5$ in men and $\geq 4$ in women ${ }^{(36)}$; high LDL-C was defined as 
levels $\geq 100 \mathrm{mg} / \mathrm{dl}^{(35)}$; high TAG was defined as levels $\geq 150 \mathrm{mg} / \mathrm{dl}^{(35)}$; high fasting blood glucose was defined as levels $\geq 100 \mathrm{mg} / \mathrm{dl}^{(37)}$; and high glycosylated $\mathrm{Hb}$ was defined as levels $\geq 7 \%{ }^{(37)}$. High hs-CRP, fibrinogen and PAI-1 were defined as levels equal to or greater than the median values in our sample. The study was approved by the Institutional Review Board of the University of Puerto Rico Medical Sciences Campus.

\section{Statistical analysis}

Sex differences for continuous and categorical variables were examined using the Student $t$ test and the $\chi^{2}$ test statistic, respectively. Three approaches were used to determine which obesity index had a stronger association with individual cardiometabolic risk factors. First, we computed Pearson's product moment correlation coefficients, controlling for age and sex, between each obesity index (BMI, WC, WHR and WHtR) and the following cardiometabolic risk factors: SBP and DBP, HDL-C, TC:HDL-C, LDL-C, TAG, fasting blood glucose, glycosylated $\mathrm{Hb}$, hs-CRP, fibrinogen and PAI-1. Second, obesity indices were divided into tertiles, and the highest tertile was designated as the high-risk group with the bottom tertile serving as the reference group. Logistic regression was used to estimate the multivariate-adjusted prevalence odds ratios (POR), with 95\% confidence intervals, between the presence of individual cardiometabolic risk factors and each obesity index, adjusting for age and sex. We defined an aggregated measure of overall cardiometabolic risk as the presence of any of the components of the metabolic syndrome, except for abdominal obesity, since WC was one of the obesity indices under examination. Third, the relative ability of the obesity indices to predict overall cardiometabolic risk was evaluated by calculating the area under the receiver-operating characteristic (ROC) curves. All analyses incorporated the sampling weight measures to obtain unbiased estimates from the complex sampling design using the STATA for Windows statistical software package version $10 \cdot 0$ (StataCorp LP, College Station, TX, USA).

\section{Results}

A total of 858 residents of the San Juan Metropolitan Area participated in the study. Study participants had a mean age of $49 \cdot 4$ (sD $16 \cdot 1)$ years; $65 \cdot 7 \%$ were women, $72 \%$ had at least 12 years of education, $67 \%$ had an annual family income <\$US20000 and 20\% were current smokers (Table 1). The distribution of annual family income, current smoking, WC, WHR, HDL-C, TC:HDL-C, TAG, SBP, DBP and fibrinogen differed significantly by $\operatorname{sex}(P<0 \cdot 05)$. Table 2 shows the prevalence of cardiometabolic risk factors in the participants. According to BMI classification, $37 \cdot 7 \%$ were overweight and $41.3 \%$ were obese, whereas $22.5 \%$ were normal weight and $1.9 \%$ were underweight; more men were overweight, whereas more women were obese $(P=0 \cdot 06)$. According to WC, $48 \cdot 6 \%$ were classified as high risk, with a greater proportion of women at high

Table 1 General characteristics of the study population

\begin{tabular}{|c|c|c|c|c|c|c|c|}
\hline \multirow[b]{2}{*}{ Characteristic } & \multicolumn{2}{|c|}{ Total $(n 858)$} & \multicolumn{2}{|c|}{ Women (n 563) } & \multicolumn{2}{|c|}{ Men ( $n$ 295) } & \multirow[b]{2}{*}{$P$ value } \\
\hline & Mean & SD & Mean & SD & Mean & SD & \\
\hline Age (years) & $49 \cdot 4$ & $16 \cdot 1$ & $49 \cdot 0$ & $15 \cdot 9$ & $50 \cdot 1$ & $16 \cdot 6$ & $>0.1$ \\
\hline$\geq 12$ years of education (\%) & \multicolumn{2}{|c|}{$71 \cdot 7$} & \multicolumn{2}{|c|}{$73 \cdot 0$} & \multicolumn{2}{|c|}{$69 \cdot 2$} & $>0 \cdot 1$ \\
\hline Annual family income $<$ \$US20 $000(\%)$ & \multicolumn{2}{|c|}{$67 \cdot 2$} & \multicolumn{2}{|c|}{$70 \cdot 2$} & \multicolumn{2}{|c|}{$61 \cdot 3$} & 0.03 \\
\hline Current smoking (\%) & \multicolumn{2}{|c|}{$19 \cdot 9$} & \multicolumn{2}{|c|}{$17 \cdot 8$} & \multicolumn{2}{|c|}{$24 \cdot 1$} & 0.02 \\
\hline BMI $\left(\mathrm{kg} / \mathrm{m}^{2}\right)$ & $29 \cdot 7$ & $6 \cdot 6$ & $29 \cdot 9$ & $7 \cdot 0$ & $29 \cdot 2$ & $6 \cdot 0$ & $>0 \cdot 1$ \\
\hline WC $(\mathrm{cm})$ & $93 \cdot 0$ & $14 \cdot 7$ & $90 \cdot 4$ & $14 \cdot 3$ & $97 \cdot 8$ & $14 \cdot 3$ & $<0.001$ \\
\hline WHR & 0.9 & $0 \cdot 1$ & $0 \cdot 8$ & $0 \cdot 1$ & 0.9 & 0.8 & $<0.001$ \\
\hline WHtR & $0 \cdot 6$ & $0 \cdot 1$ & 0.6 & 0.9 & 0.6 & 0.8 & $>0 \cdot 1$ \\
\hline HDL-C (mg/dl) & $49 \cdot 3$ & $13 \cdot 0$ & $51 \cdot 3$ & $12 \cdot 2$ & $45 \cdot 6$ & $13 \cdot 6$ & $<0.001$ \\
\hline TC:HDL-C & $4 \cdot 1$ & $1 \cdot 3$ & 3.9 & $1 \cdot 0$ & $4 \cdot 4$ & $1 \cdot 8$ & $<0.001$ \\
\hline LDL-C (mg/dl)† & $117 \cdot 5$ & $39 \cdot 1$ & $116 \cdot 4$ & $35 \cdot 4$ & $119 \cdot 7$ & $45 \cdot 3$ & $>0 \cdot 1$ \\
\hline TAG $(\mathrm{mg} / \mathrm{dl}) \dagger$ & $141 \cdot 7$ & $106 \cdot 5$ & $128 \cdot 6$ & $83 \cdot 9$ & $167 \cdot 0$ & $136 \cdot 6$ & $<0.001$ \\
\hline $\mathrm{SBP}(\mathrm{mmHg}) \dagger$ & $120 \cdot 1$ & $21 \cdot 1$ & $116 \cdot 8$ & $20 \cdot 5$ & $126 \cdot 3$ & $20 \cdot 8$ & $<0.001$ \\
\hline $\mathrm{DBP}(\mathrm{mmHg}) \dagger$ & $73 \cdot 0$ & $11 \cdot 1$ & $71 \cdot 0$ & $10 \cdot 6$ & $76 \cdot 6$ & $11 \cdot 1$ & $<0.001$ \\
\hline Fasting blood glucose $(\mathrm{mg} / \mathrm{dl}) \ddagger$ & $113 \cdot 4$ & $48 \cdot 3$ & $111 \cdot 6$ & $50 \cdot 3$ & $116 \cdot 9$ & $44 \cdot 1$ & $>0 \cdot 1$ \\
\hline Glycosylated $\mathrm{Hb}(\%) \S$ & $6 \cdot 3$ & $1 \cdot 5$ & $6 \cdot 3$ & $1 \cdot 6$ & $6 \cdot 4$ & $1 \cdot 5$ & $>0 \cdot 1$ \\
\hline Fibrinogen (mg/dl)\| & $321 \cdot 5$ & $77 \cdot 8$ & $328 \cdot 7$ & $73 \cdot 9$ & $307 \cdot 9$ & $83 \cdot 2$ & $<0.001$ \\
\hline hs-CRP (mg/l) & 0.6 & 0.9 & 0.6 & $0 \cdot 8$ & 0.4 & 0.8 & 0.08 \\
\hline PAI-1 (IU/ml)+† & $18 \cdot 9$ & $2 \cdot 9$ & $15 \cdot 2$ & $2 \cdot 61$ & $25 \cdot 8$ & $6 \cdot 6$ & 0.08 \\
\hline
\end{tabular}

WC, waist circumference; WHR, waist-to-hip ratio; WHtR, waist-to-height ratio; HDL-C, HDL cholesterol; TC, total cholesterol; LDL-C, LDL cholesterol; SBP, systolic blood pressure; DBP, diastolic blood pressure; hs-CRP, high-sensitivity C-reactive protein; PAI-1, plasminogen activator inhibitor-1.

*Student's $t$ test or the $\chi^{2}$ test.

tMissing two participants.

‡Missing one participant.

\$Missing three participants.

\|Missing eleven participants.

-Missing seventeen participants.

t+Missing forty-nine participants. 
Table 2 Prevalence of cardiometabolic risk factors in the study group

\begin{tabular}{|c|c|c|c|c|}
\hline Risk factor & Total $(n 858)$ & Women ( $n$ 563) & Men $(n$ 295) & $P$ value ${ }^{\star}$ \\
\hline \multicolumn{5}{|l|}{ High BMI (kg/m²) } \\
\hline Overweight & $37 \cdot 7$ & $35 \cdot 2$ & $42 \cdot 3$ & 0.060 \\
\hline Obese & $41 \cdot 3$ & $44 \cdot 1$ & $36 \cdot 1$ & \\
\hline High risk by WC & $48 \cdot 6$ & $54 \cdot 4$ & $37 \cdot 6$ & $<0.001$ \\
\hline High risk by WHR & $33 \cdot 7$ & $41 \cdot 6$ & $18 \cdot 6$ & $<0.001$ \\
\hline High risk by WHtR & $80 \cdot 3$ & $78 \cdot 5$ & $83 \cdot 7$ & 0.068 \\
\hline High TC:HDL-C & $35 \cdot 3$ & $39 \cdot 6$ & $27 \cdot 1$ & $<0.001$ \\
\hline High LDL-C & $64 \cdot 6$ & $64 \cdot 1$ & 65.9 & $>0 \cdot 1$ \\
\hline Low HDL-C & $45 \cdot 8$ & $51 \cdot 7$ & $34 \cdot 6$ & $<0.001$ \\
\hline High TAG & $31 \cdot 2$ & $26 \cdot 8$ & $39 \cdot 7$ & $<0.001$ \\
\hline High blood pressure & $45 \cdot 6$ & $40 \cdot 1$ & $55 \cdot 9$ & $<0.001$ \\
\hline High fasting blood glucose & $49 \cdot 8$ & $43 \cdot 9$ & $61 \cdot 0$ & $<0.001$ \\
\hline High glycosylated $\mathrm{Hb}$ & $15 \cdot 2$ & $13 \cdot 6$ & $18 \cdot 4$ & 0.062 \\
\hline
\end{tabular}

WC, waist circumference; WHR, waist-to-hip ratio; WHtR, waist-to-height ratio; TC, total cholesterol; HDL-C, HDL cholesterol; LDL-C, LDL cholesterol.

${ }^{*}$ The $\chi^{2}$ test.

Table 3 Partial Pearson's correlations of cardiometabolic risk factors and obesity indices (controlling for age and sex)

\begin{tabular}{|c|c|c|c|c|c|c|c|c|c|c|c|}
\hline Obesity index & SBP & DBP & HDL-C & TC:HDL-C & LDL-C & TAG & $F G$ & $\mathrm{HbA1c}$ & hs-CRP & Fibrinogen & PAI-1 \\
\hline W & 0.27 & 0.36 & $-0 \cdot 24^{\star * *}$ & $0.27^{\star \star \star}$ & $1^{*}$ & 0.17 & $0 \cdot 2$ & 0.2 & $0 \cdot 23^{\star \star \star}$ & $0 \cdot 1$ & $0 \cdot 12^{*}$ \\
\hline WHR & $0 \cdot 17^{\star \star \star}$ & $0 \cdot 18^{\star \star \star}$ & $-0 \cdot 19^{\star \star \star}$ & $0 \cdot 26^{\star \star \star}$ & $0 \cdot 11^{*}$ & $0 \cdot 21^{\star \star \star}$ & $0 \cdot 29^{\star \star \star}$ & $0 \cdot 28^{\star \star \star}$ & $0 \cdot 15^{\star \star \star}$ & $0 \cdot 13^{\star \star \star}$ & $0 \cdot 13^{\star \star \star}$ \\
\hline $\mathrm{BMI}$ & $0 \cdot 26^{\star \star \star}$ & $0 \cdot 35^{\star \star *}$ & $-0 \cdot 18^{\star \star \star}$ & $0.21^{\star \star \star}$ & $0 \cdot 10^{*}$ & $0 \cdot 13^{\star \star \star}$ & $0 \cdot 22^{\star \star \star}$ & $0 \cdot 20^{\star \star \star}$ & $0.24^{\star \star \star}$ & 0. & 0.09 \\
\hline WHtR & $0 \cdot 25^{\star \star \star}$ & $0 \cdot 34^{\star \star \star}$ & $-0 \cdot 23^{\star \star \star}$ & $0 \cdot 26^{\star * \star}$ & $0 \cdot 12^{*}$ & $0 \cdot 18^{\star \star \star}$ & $0 \cdot 28^{\star \star \star}$ & $0 \cdot 26^{\star \star \star}$ & $0 \cdot 24^{\star \star \star}$ & $0 \cdot 19^{\star \star \star}$ & $0 \cdot 12^{\star}$ \\
\hline
\end{tabular}

SBP, systolic blood pressure; DBP, diastolic blood pressure; HDL-C, HDL cholesterol; TC, total cholesterol; LDL-C, LDL cholesterol; FG, fasting blood glucose; $\mathrm{HbA1c}$, glycosylated $\mathrm{Hb}$; hs-CRP, high-sensitivity C-reactive protein; PAl-1, plasminogen activator inhibitor; WC, waist circumference; WHR, waist-to-hip ratio; WHtR, waist-to-height ratio.

${ }^{*} P<0.05 ;{ }^{* \star *} P<0.001$.

risk $(P<0 \cdot 001)$. According to WHR, 33.7\% were classified as high risk, with a greater proportion of women at high risk $(P<0 \cdot 001)$. According to WHtR, $80.3 \%$ were classified as high risk, which was marginally higher in men than in women $(P=0.068)$. The prevalence of dyslipidaemia was high in this sample; high TC:HDL-C was $35 \cdot 3 \%$, high LDL-C was $64 \cdot 6 \%$, low HDL-C was $45 \cdot 8 \%$ and high TAG was $31 \cdot 2 \%$, with generally higher prevalence in women $(P<0 \cdot 001)$. The prevalence of high blood pressure was $45 \cdot 6 \%$, that of high fasting glucose levels $(\geq 100 \mathrm{mg} / \mathrm{dl})$ was $49 \cdot 8 \%$ and of high glycosylated $\mathrm{Hb}(\geq 7 \cdot 0 \%$ ) was $15 \cdot 2 \%$ (Table 2 ).

Table 3 shows the partial correlation analyses using Pearson's coefficients between the obesity indices (WC, WHR, WHtR and BMI) and cardiometabolic risk factors under study, controlling for age and sex because of significant sex differences between these variables. All four obesity indices were significantly $(P<0 \cdot 05)$ correlated with blood pressure, HDL-C, TC:HDL-C, LDL-C, TAG, fasting blood glucose, glycosylated $\mathrm{Hb}$, hs-CRP, fibrinogen and PAI-1, except for the correlation between PAI-1 and BMI. For HDL-C, SBP and DBP and PAI-1, WC had the highest correlation coefficient. However, the correlation for HDL-C was in the opposite direction compared with the other cardiometabolic factors. For TC:HDL-C, all indices had similar coefficients, except BMI, which had the lowest. For LDL-C, WHtR had the highest coefficient. For TAG, fasting blood glucose and glycosylated $\mathrm{Hb}$,
WHR had the highest coefficients. For fibrinogen, WC and WHtR had the highest coefficients, whereas for hs-CRP, BMI and WHtR had the highest coefficients.

Figure 1 shows the adjusted POR for high LDL-C and TC:HDL-C, low HDL-C, high TAG and high blood pressure by obesity index categorized by tertiles. The tertiles for each obesity index were: BMI (tertile $1,<26.4 \mathrm{~kg} / \mathrm{m}^{2}$; tertile $2,26 \cdot 4-31 \cdot 2 \mathrm{~kg} / \mathrm{m}^{2}$; tertile $3,>31 \cdot 2 \mathrm{~kg} / \mathrm{m}^{2}$ ); WC (tertile 1, $<86.4 \mathrm{~cm}$; tertile 2, 86.4-98.5 cm; tertile 3, $>98.5 \mathrm{~cm}$ ); WHR (tertile $1,<0.82$; tertile $2,0.82-0.91$; tertile 3,>0.91); and WHtR (tertile $1,<0.54$; tertile 2, $0 \cdot 54-0 \cdot 61$; tertile $3,>0 \cdot 61$ ). For high LDL-C, WC was the only index that significantly increased the POR at tertile 2 , although all four obesity indices significantly increased the POR at tertile 3 compared with tertile $1(P<0 \cdot 05)$; BMI had the lowest POR at all points. For low HDL-C, WC had the highest POR at all points, whereas WHR had the lowest POR at tertile 2 and BMI at tertile $3(P<0 \cdot 05)$. However, all four obesity indices significantly increased the POR at tertiles 2 and 3 compared with tertile 1, except for WHR at tertile $2(P<0 \cdot 05)$. For high TC:HDL-C, WHtR had the highest POR, whereas BMI had the lowest, although all four obesity indices significantly increased the POR at tertiles 2 and 3 compared with tertile 1 $(P<0 \cdot 05)$. For high TAG, WHR had the highest POR and BMI the lowest, although all four obesity indices significantly increased the POR at tertiles 2 and 3 compared with tertile $1(P<0 \cdot 05)$. For high blood pressure, WHtR 
High LDL-C

$(\geq 130 \mathrm{mg} / \mathrm{dl})$

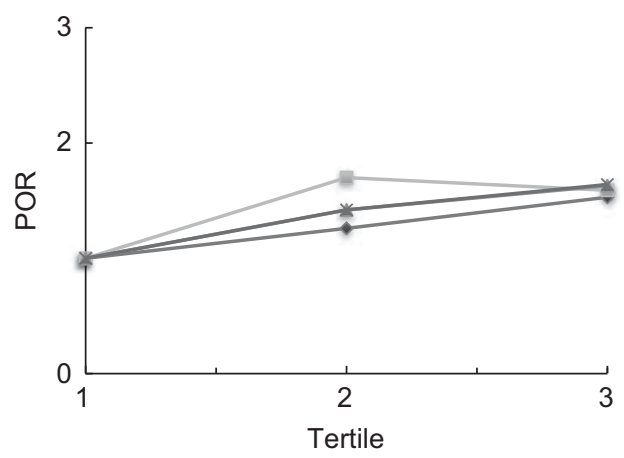

High TC:HDL-C

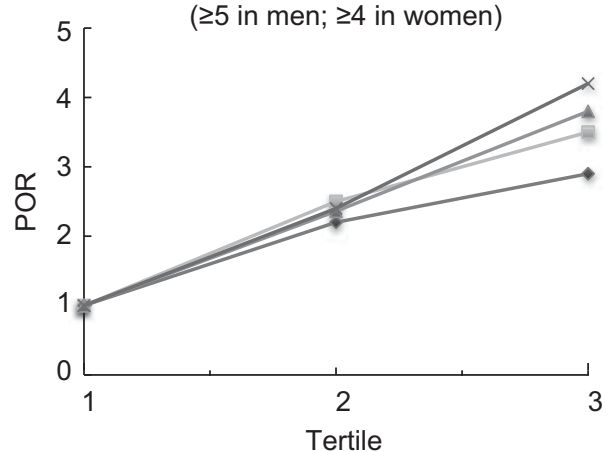

Low HDL-C

( $\leq 40 \mathrm{mg} / \mathrm{dl}$ in men; $\leq 50 \mathrm{mg} / \mathrm{dl}$ in women)
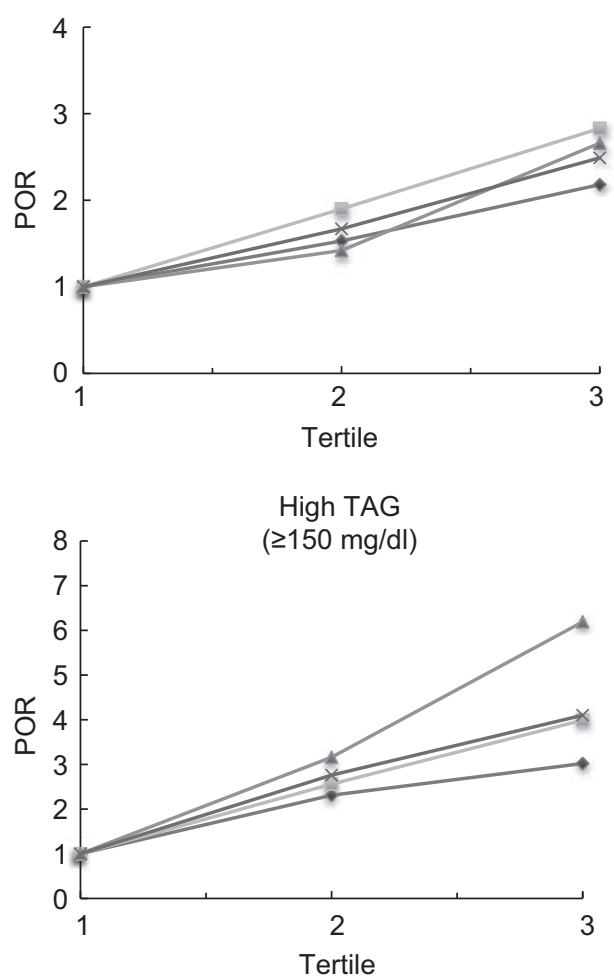

High blood pressure (systolic $\geq 130 /$ diastolic $\geq 85 \mathrm{mmHg}$ )

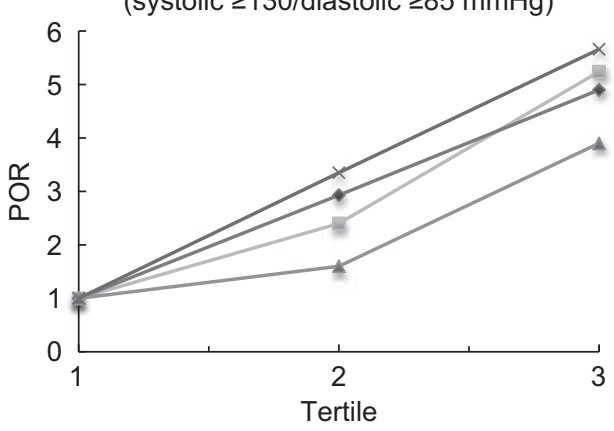

Fig. 1 Prevalence odds ratio (POR) of high LDL cholesterol (LDL-C), low HDL cholesterol (HDL-C), high total cholesterol (TC):HDL-C, high TAG and high blood pressure by BMI $(--)$, waist circumference (WC; $-\square$ - , waist-to-hip ratio (WHR; - and waist-to-height ratio (WHtR; $\rightarrow \leftarrow$ ), adjusted by age and sex. POR (95\% Cl) for high LDL-C: WC (tertile 2, 1.70 (1.19, 2.43); tertile 3, $1.59(1 \cdot 10,2 \cdot 28)$ ), WHR (tertile 3, 1.63 (1.06, 2.53)), WHtR (tertile 3, 1.64 (1.14, 2.36)) and BMI (tertile 3, 1.53 (1.07, 2.18)). POR (95\% Cl) for low HDL-C: WC (tertile 2, $1.90(1 \cdot 33,2 \cdot 72)$; tertile 3, $2 \cdot 83(1 \cdot 96,4 \cdot 09))$, WHR (tertile 2, 1.42 (0.99, 2.02); tertile $3,2 \cdot 66(1 \cdot 72,4 \cdot 11))$, WHtR (tertile 2, $1 \cdot 67(1 \cdot 17,2 \cdot 37)$; tertile $3,2 \cdot 49(1 \cdot 74,3 \cdot 58)$ ) and BMI (tertile 2, 1.53 $(1 \cdot 08,2 \cdot 16)$; tertile 3 , $2 \cdot 18(1 \cdot 54,3 \cdot 10))$. POR $(95 \% \mathrm{Cl})$ for high TC:HDL-C: WC (tertile 2, $2 \cdot 55(1 \cdot 73,3 \cdot 74)$; tertile 3, $2 \cdot 52(2 \cdot 39,5 \cdot 20)$ ), WHR (tertile 2, $2 \cdot 36$ (1.51, 3.21); tertile 3, $2.92(2 \cdot 01,3 \cdot 44)$ ), WHtR (tertile 2, $2 \cdot 40(1 \cdot 62,3 \cdot 54)$; tertile $3,4 \cdot 20(2 \cdot 84,6 \cdot 20)$ ) and BMI (tertile 2, 2.20 (1.51, 3.21); tertile 3, $2.92(2.01,4.24))$. POR (95\% Cl) for high TAG: WHR (tertile 2, $3.16(2 \cdot 02,4.94)$; tertile 3, 6.19 (3.78, 10.16)), WC (tertile 2, $2.56(1 \cdot 68,3 \cdot 89)$; tertile 3, 3.99 (2.64, 6.03)), WHtR (tertile 2, $2 \cdot 76(1 \cdot 81,4 \cdot 21)$; tertile 3, 4.10 (2.69, 6.23)) and BMI (tertile 2, $2.31(1.55,3 \cdot 45)$; tertile 3, 3.02 (2.03, 4.51)). POR (95\% Cl) for high blood pressure: WHtR (tertile 2, 3.35 (2.19, $5 \cdot 13)$; tertile 3, 5.66 (3.69, 8.69)), WC (tertile 2, 2.39 (1.58, 3.64); tertile 3, 5.24 (3.41, 8.06)), BMI (tertile 2, 2.93 (1.92, 4.46); tertile 3, $4.90(3 \cdot 19,7.54))$ and WHR (tertile 2, $1.60(1 \cdot 06,2 \cdot 42)$; tertile 3, 3.91 $(2 \cdot 41,6 \cdot 34))$

had the highest POR and WHR had the lowest, although all four obesity indices significantly increased the POR at tertiles 2 and 3 compared with tertile $1(P<0 \cdot 05)$.

The adjusted POR estimates for high fasting glucose and high glycosylated $\mathrm{Hb}$ according to the four obesity indices are shown in Fig. 2. For high fasting glucose, WC had the highest POR, whereas BMI had the lowest, although all four obesity indices significantly increased the POR at tertiles 2 and 3 compared with tertile 1 $(P<0 \cdot 05)$. For high glycosylated Hb, WHR was superior 

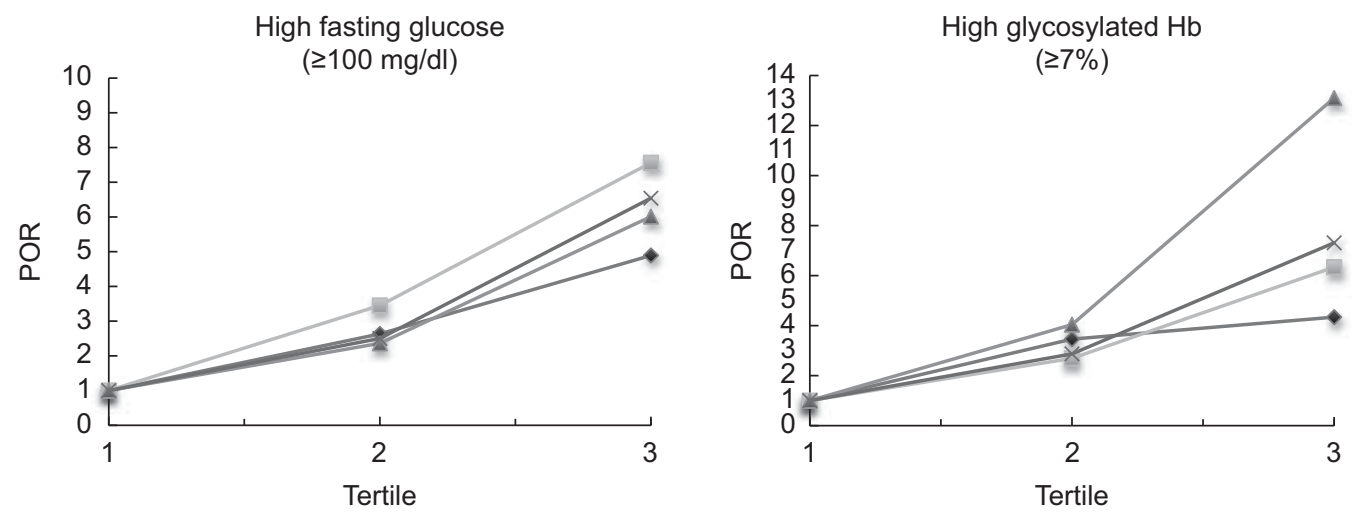

Fig. 2 Prevalence odds ratio (POR) of high fasting glucose and high glycosylated $\mathrm{Hb}$ by $\mathrm{BMI}(--)$, waist circumference (WC; —- ) , waist-to-hip ratio (WHR; - - ) and waist-to-height ratio (WHtR; $\leftarrow$ ), adjusted by age and sex. POR $(95 \% \mathrm{Cl})$ for high fasting glucose: WC (tertile 2, 3.45 (2.34, 5.08); tertile 3, 7.56 (5.05, 11.33)), WHR (tertile 2, $2 \cdot 36(1 \cdot 62,3 \cdot 44)$; tertile 3, 6.01 $(3 \cdot 80,9 \cdot 50)$ ), WHtR (tertile 2, $2 \cdot 50(1 \cdot 72,3 \cdot 67)$; tertile $3,6 \cdot 54(4 \cdot 40,9 \cdot 73)$ ) and BMI (tertile 2, $2 \cdot 63(1 \cdot 80,3 \cdot 83)$; tertile 3, 4.89 (3.31, 7.21)). POR (95\% Cl) for high glycosylated Hb: WHR (tertile 2, 4.04 (1·79, 9.09); tertile 3, $13 \cdot 10(5 \cdot 69$, 30·16)), WC (tertile 2, 2.69 $(1 \cdot 36,5 \cdot 30)$; tertile $3,6 \cdot 34(3 \cdot 30,12 \cdot 17))$, WHtR (tertile $2,2 \cdot 87(1 \cdot 39,5 \cdot 96)$; tertile $3,7 \cdot 31(3 \cdot 64,14 \cdot 67))$ and BMI (tertile $2,3 \cdot 46$ $(1 \cdot 88,6 \cdot 37)$; tertile $3,4 \cdot 34(2 \cdot 33,8 \cdot 09))$
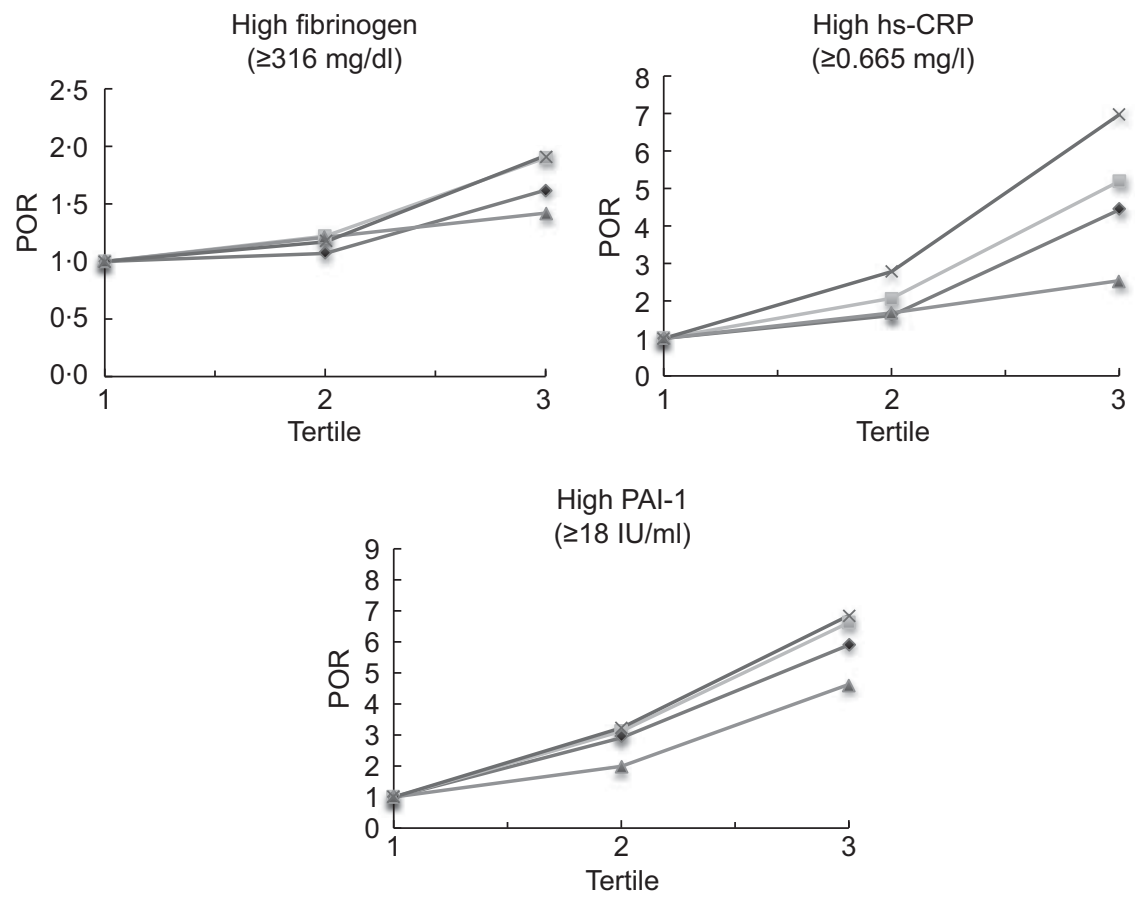

Fig. 3 Prevalence odds ratio (POR) of high fibrinogen, high plasminogen activator inhibitor-1 (PAl-1) and high high-sensitivity C-reactive protein (hs-CRP) by BMI (- - ), waist circumference (WC; - - ), waist-to-hip ratio (WHR; - ) and waist-toheight ratio (WHtR; $\rightarrow-$ ), adjusted by age and sex. POR $(95 \% \mathrm{Cl})$ for high fibrinogen: WC (tertile $2,1 \cdot 22(0 \cdot 86,1 \cdot 73)$; tertile 3 , $1 \cdot 90(1 \cdot 33,2 \cdot 71)$ ), WHtR (tertile 2, 1·17, 0.83, 1.66; tertile 3, 1.92 (1.34, 2.70)), BMI (tertile 2, 1.07, 0.76, 1.50; tertile 3, 1.62 (1.15, 2.29)) and WHR (tertile 2, 1.21, 0.85, 1.73; tertile 3, 1.42 (0.93, 2.16)). POR (95\% Cl) for high PAI-1: WHtR (tertile 2, 3.23 (2.22, 4.69); tertile 3, $6 \cdot 85(4 \cdot 62,10 \cdot 17)$ ), WC (tertile 2, 3.12 (2.14, 4.55); tertile 3, 6.62 (4.46, 9.82)), BMI (tertile 2, 2.91 (2.01, 4.21); tertile 3, 5.90 (4.04, 8.62)) and WHR (tertile 2, $1.99(1 \cdot 38,2 \cdot 86)$; tertile 3, 4.63 (2.95, 7.28)). POR (95\% Cl) for high hs-CRP: WHtR (tertile 2, $2 \cdot 78(1 \cdot 48,5 \cdot 24)$; tertile $3,6 \cdot 97(3 \cdot 86,12 \cdot 58)$ ), WC (tertile 2, $2 \cdot 07(1 \cdot 14,3 \cdot 57)$; tertile $3,5 \cdot 19(3 \cdot 06,8 \cdot 81)$ ), WHR (tertile 2, $1.68(1 \cdot 02,2 \cdot 77)$; tertile $3,2 \cdot 54(1 \cdot 44,4 \cdot 49))$ and BMI (tertile 2, $1 \cdot 61(0 \cdot 89,2 \cdot 93)$; tertile 3, $4 \cdot 43(2 \cdot 61,7 \cdot 51)$ )

to the other indices, particularly at tertile 3, whereas BMI had the lowest, although all four obesity indices significantly increased the POR at tertiles 2 and 3 compared with tertile $1(P<0 \cdot 05)$.
Figure 3 shows the POR for high fibrinogen, high PAI-1 and high hs-CRP according to the four obesity indices. For fibrinogen, both WC and WHtR had the highest POR, whereas WHR had the lowest in tertile 3, which was not 
significant; no index significantly increased the POR at tertile $2(P<0 \cdot 05)$. For high PAI-1, both WC and WHtR had the highest POR, whereas WHR had the lowest, although all four obesity indices significantly increased the POR at tertiles 2 and 3 compared with tertile 1 $(P<0 \cdot 05)$. For high hs-CRP, WHtR had the highest POR, whereas WHR had the lowest, although all four obesity indices significantly increased the POR at tertiles 2 and 3 compared with tertile 1 , except BMI in tertile $2(P<0 \cdot 05)$.

Figure 4 shows the POR for overall cardiometabolic risk according to the four obesity indices. The highest POR at tertile 3 was for WHR, followed by WC and WHtR, and the lowest was for BMI. However, all four obesity indices significantly increased the POR at tertiles 2 and 3 compared with tertile $1(P<0 \cdot 05)$.

Results were similar after additional adjustment for education and smoking status and when underweight individuals were excluded from these models ( $n 16 ; 1.9 \%$ of the sample; data not shown).

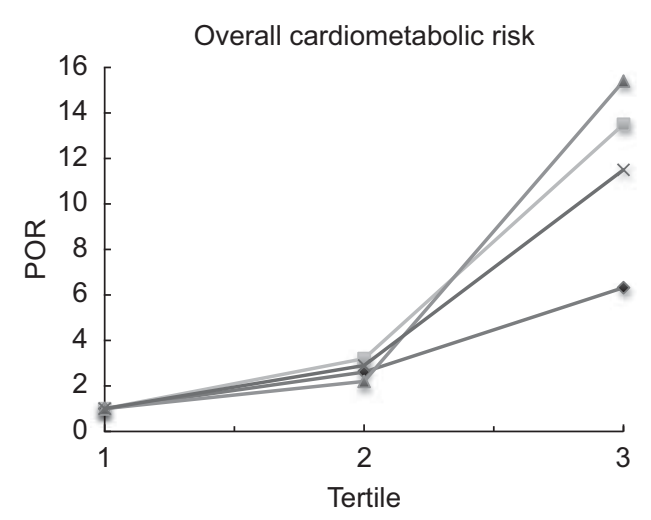

Fig. 4 Prevalence odds ratio (POR) of the overall cardiometabolic risk (one or more risk factors) by BMI $(-)$ ), waist circumference (WC; - - ) , waist-to-hip ratio (WHR; - ) and waist-to-height ratio (WHtR; $\rightarrow \leftarrow$ ), adjusted by age and sex. POR $(95 \% \mathrm{Cl})$ of overall cardiometabolic risk: WC (tertile 2, 3.21 (2.09, 4.94); tertile 3, 13.53 (6.95, 26.30)), WHR (tertile 2, 2.90 (1.91, 4.41); tertile 3, 11.50 (6.09, 21.71)), WHtR (tertile 2, 2.21 (1.45, 3.37); tertile 3, 15.41 (7.10, 33.43)) and BMI (tertile 2, $2 \cdot 61(1 \cdot 70,4 \cdot 01)$; tertile $3,6 \cdot 32(3 \cdot 82,10 \cdot 48)$ )

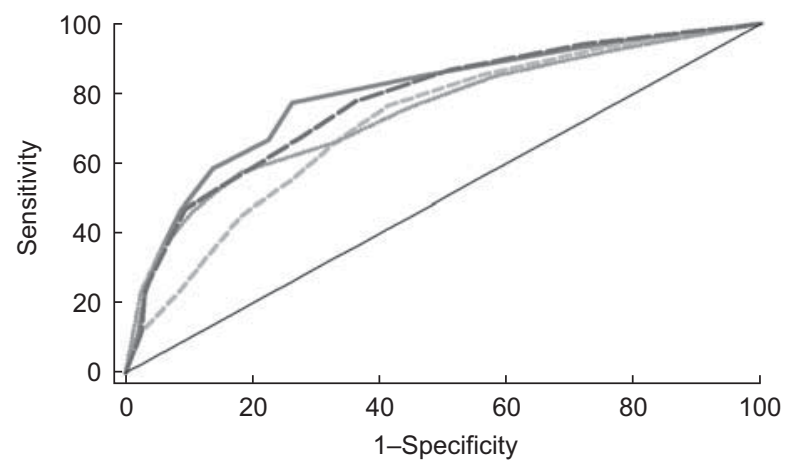

Fig. 5 Receiver-operating characteristic curves for overall cardiometabolic risk according to BMI (-----), waist circumference $(-)$, waist-to-hip ratio $(-)$ and waist-to-height ratio
Figure 5 shows ROC curves for all obesity indices in relation to the overall cardiometabolic risk. BMI had the lowest sensitivity and specificity for cardiometabolic risk compared with the other obesity indices.

\section{Discussion}

The present study conducted in adults living in Puerto Rico found that all four obesity indices (BMI, WC, WHR and WHtR) correlated significantly with blood pressure, HDL-C, TC:HDL-C, LDL-C, TAG, fasting blood glucose, glycosylated $\mathrm{Hb}$, hs-CRP, fibrinogen and PAI-1. The present study also showed differences in the obesity index that best correlated with cardiometabolic risk factors. WHtR had the highest POR for high TC:HDL-C, high blood pressure, high hs-CRP, fibrinogen and PAI-1; WC had the highest POR for low HDL-C and LDL-C and high fasting blood glucose; and WHR had the highest POR for the overall cardiometabolic risk, for high TAG and high glycosylated $\mathrm{Hb}$. BMI was consistently the index with the lowest POR for most cardiometabolic risk factors, although the POR increased significantly as BMI tertiles increased, and with the smallest area under the curve for overall cardiometabolic risk.

Earlier studies have shown that individuals with greater WC and WHR have an increased risk of mortality due to $\mathrm{CHD}^{(38,39)}$ and $\mathrm{CVD}^{(40,41)}$. Therefore, several studies have been conducted to determine which index is the strongest predictor of disease risk, in order to easily identify these individuals in the clinical or public health setting. Traditionally, BMI has been used; however, some studies show that individuals may be categorized as normal or overweight by BMI but at the same time as obese or high risk by other body composition measures such as WC, WHR or percentage body fat ${ }^{(42)}$, with ethnic differences ${ }^{(43)}$. Indeed, several studies have shown that WC or WHR is a better indicator of cardiometabolic risk factors compared with BMI among Caucasians, African Americans and Asians ${ }^{(6,8,44)}$. In addition, the Latin American Consortium of Studies in Obesity also found that WHR was the most accurate index to screen for high-risk CHD (using the Framingham equation to estimate the expected 10-year risk) in 18976 Latin Americans compared with WC and BMI, with better performance in whites ${ }^{(27)}$. Therefore, fat distribution appears to be an important measure of cardiometabolic disease risk and should be taken into account in the clinical and public health settings in addition to body mass measures.

The present study showed that the four obesity indices correlated significantly with all of the cardiometabolic risk factors. Although the strength of the correlations was not high, it was lowest for LDL-C, fibrinogen and PAI and highest for DBP. High blood pressure is easily affected by obesity, as there are several mechanisms through which obesity directly causes hypertension, such as activation 
of the sympathetic nervous system, changes in arterialpressure control mechanism of diuresis and natriuresis, $\mathrm{Na}$ retention with extracellular-fluid volume expansion, increase in levels of plasma renin activity, angiotensinogen, angiotensin II and aldosterone and altered vascular function via insulin resistance and inflammation ${ }^{(45)}$. Others have found similar correlations between these indices and several cardiometabolic risk factors ${ }^{(8,21)}$. In addition, although the present study showed that WC, WHR or WHtR may be a better predictor of cardiometabolic risk compared with BMI, the latter was also found to be significantly associated with most cardiometabolic risk factors. Other studies have also found WC, WHR or WHtR to be a stronger predictor of disease risk, while also showing that all four obesity indices significantly predict these in Caucasians and Asians ${ }^{(8,10-14,17,20,21,42,46)}$.

The present study also failed to detect a single obesity index that best predicted all cardiometabolic risk factors. Several studies have found WHtR to be superior to the other indices in predicting these risk factors ${ }^{(13,16,17,17)}$, including a meta-analysis of ten studies mainly in Asians ${ }^{(15)}$ '. However, the present study found that WHtR was as good at predicting cardiometabolic risk factors as WC and WHR, similar to the study by Park et $a l^{(9)}$ in Koreans.

In terms of practicality issues, WC and WHtR are the easiest and quickest assessment methods in the clinical setting, without the need to have a calibrated balance or to take off clothes to accurately measure weight for BMI. In addition, the calculation is easier than for BMI. However, WC does not take into account stature, and studies show that individuals of shorter stature have higher body fat compared with taller individuals ${ }^{(47)}$. WHR also is a fairly good cardiometabolic disease index, although the HC is not always taken accurately since it is often difficult to ascertain the morphological points to assess this measure; therefore, the measurement errors of both methods may be adding up. In addition, proportional changes in WC and HC may not change WHR despite changes in body size, whereas WHtR changes only when WC decreases or increases. Therefore, WHtR may be the easiest index while also being more sensitive to changes in body composition. However, WHtR is not widely used in studies or in the clinical setting. The small differences in its discriminatory capability in cardiometabolic risk factors may be of limited clinical relevance and possibly the reason for not being adopted universally.

It has been proposed that an excess of central adiposity leads to several metabolic processes that increase cardiometabolic risk factors. These are: (i) exposing the liver to high concentrations of free fatty acids, which may then impair several hepatic metabolic processes leading to hyperinsulinaemia, glucose intolerance and hypertriacylglycerolaemia; (ii) increased production of adipokines and inflammatory cytokines, which also contributes to the insulin-resistant state, as well as to a state of inflammation; and (iii) impaired clearance and storage of fat, which then accumulates in certain organs such as the liver, heart and pancreas ${ }^{(48)}$.

One of the strengths of the study was inclusion of a representative sample of Spanish-speaking adults in the San Juan Metropolitan Area, which could be considered a Hispanic sample of homogeneous origin. In addition, the measurements were taken by trained professionals using standarized anthropometric procedures with extensive data from laboratory measurements on cardiovascular risk factors in both men and women aged 21-79 years. Our study has limitations that should be considered. This was a cross-sectional study; therefore, it should be interpreted with caution. Prospective studies are needed to better elucidate the obesity index that best predicts future cardiometabolic conditions. In addition, although four obesity indicators were used, we did not include a direct measure of body composition, which could provide more accurate information. However, such measurements are difficult to take in large epidemiological studies.

In conclusion, in this representative sample of adults living in the San Juan Metropolitan Area, as the obesity indices increased, the cardiometabolic risk factors also increased significantly. In addition, as tertiles of obesity indices increased, the POR estimates for cardiometabolic risk factors were found to be significantly higher. However, indices of body fat centralization (WC, WHR and $\mathrm{WHtR}$ ) appeared to be better predictors of cardiometabolic risk factors compared with BMI.

\section{Acknowledgements}

This project was fully funded by an unrestricted grant from Merck Sharp \& Dohme (I.A.) Corporation with additional support by 1U54RR026139-01A1 grant from the National Center for Research Resources (NCRR) of the National Institutes of Health. The authors have no conflict of interest to declare. C.M.P., A.P.O. and E.S. contributed to the study design; C.M.P. and E.S. were responsible for obtaining funding; C.M.P., E.S., A.P.O. and M.G. contributed to data collection and on-site study management; C.P. and C.M.P. contributed to data analysis; and C.P., C.M.P. and A.A. contributed to manuscript preparation.

\section{References}

1. Expert Panel on the Identification, Evaluation, and Treatment of Overweight in Adults (1998) Clinical guidelines on the identification, evaluation, and treatment of overweight and obesity in adults: executive summary. Am J Clin Nutr 68, 899-917.

2. Goran MI \& Gower BA (1999) Relation between visceral fat and disease risk in children and adolescents. Am J Clin Nutr 70, Suppl. 1, 149S-156S.

3. Snijder MB, Visser M, Dekker JM et al. (2002) The prediction of visceral fat by dual-energy X-ray absorptiometry in the elderly: a comparison with computed tomography and anthropometry. Int J Obes Relat Metab Disord 26, 984-993. 
4. Lee SY \& Gallagher D (2008) Assessment methods in human body composition. Curr Opin Clin Nutr Metab Care 11, 566-572.

5. Mason C, Craig CL \& Katzmarzyk PT (2008) Influence of central and extremity circumferences on all-cause mortality in men and women. Obesity (Silver Spring) 16, 2690-2695.

6. Van Pelt RE, Evans EM, Schechtman KB et al. (2001) Waist circumference vs body mass index for prediction of disease risk in postmenopausal women. Int J Obes Relat Metab Disord 25, 1183-1188.

7. Shen W, Punyanitya M, Chen J et al. (2007) Visceral adipose tissue: relationships between single slice areas at different locations and obesity-related health risks. Int J Obes (Lond) 31, 763-769.

8. Nakamura Y, Turin TC, Kita Y et al. (2007) Associations of obesity measures with metabolic risk factors in a community-based population in Japan. Circ J 71, 776-781.

9. Park S, Choi S, Lee K et al. (2009) Waist circumference and waist-to-height ratio as predictors of cardiovascular disease risk in Korean adults. Circ J 73, 1643-1650.

10. Sung KC, Ryu S \& Reaven GM (2007) Relationship between obesity and several cardiovascular disease risk factors in apparently healthy Korean individuals: comparison of body mass index and waist circumference. Metabolism 56, 297-303.

11. Taylor AE, Ebrahim S, Ben-Shlomo Y et al. (2010) Comparison of the associations of body mass index and measures of central adiposity and fat mass with coronary heart disease, diabetes, and all-cause mortality: a study using data from 4 UK cohorts. Am J Clin Nutr 91, 547-556.

12. Christian AH, Mochari H \& Mosca LJ (2009) Waist circumference, body mass index, and their association with cardiometabolic and global risk. J Cardiometab Syndr 4, 12-19.

13. Gelber RP, Gaziano JM, Orav EJ et al. (2008) Measures of obesity and cardiovascular risk among men and women. J Am Coll Cardiol 52, 605-615.

14. Schneider HJ, Glaesmer H, Klotsche J et al. (2007) Accuracy of anthropometric indicators of obesity to predict cardiovascular risk. J Clin Endocrinol Metab 92, 589-594.

15. Lee CM, Huxley RR, Wildman RP et al. (2008) Indices of abdominal obesity are better discriminators of cardiovascular risk factors than BMI: a meta-analysis. I Clin Epidemiol 61, 646-653.

16. Can AS, Bersot TP \& Gonen M (2009) Anthropometric indices and their relationship with cardiometabolic risk factors in a sample of Turkish adults. Public Health Nutr 12, 538-546.

17. Mellati AA, Mousavinasab SN, Sokhanvar S et al. (2009) Correlation of anthropometric indices with common cardiovascular risk factors in an urban adult population of Iran: data from Zanjan Healthy Heart Study. Asia Pac J Clin Nutr 18, 217-225.

18. Ashwell M, Cole TJ \& Dixon AK (1996) Ratio of waist circumference to height is strong predictor of intraabdominal fat. BMJ 313, 559-560.

19. Browning LM, Hsieh SD \& Ashwell M (2010) A systematic review of waist-to-height ratio as a screening tool for the prediction of cardiovascular disease and diabetes: 0.5 could be a suitable global boundary value. Nutr Res Rev $\mathbf{2 3}$, 247-269.

20. Zhou Z, Hu D \& Chen J (2009) Association between obesity indices and blood pressure or hypertension: which index is the best? Public Health Nutr 12, 1061-1071.

21. Esmaillzadeh A, Mirmiran P \& Azizi F (2004) Waist-to-hip ratio is a better screening measure for cardiovascular risk factors than other anthropometric indicators in Tehranian adult men. Int J Obes Relat Metab Disord 28, 1325-1332.
22. Nyamdorj R, Qiao Q, Soderberg S et al. (2009) BMI compared with central obesity indicators as a predictor of diabetes incidence in Mauritius. Obesity (Silver Spring) 17, 342-348.

23. Ashwell M \& Hsieh SD (2005) Six reasons why the waistto-height ratio is a rapid and effective global indicator for health risks of obesity and how its use could simplify the international public health message on obesity. Int J Food Sci Nutr 56, 303-307.

24. Ghandehari H, Le V, Kamal-Bahl S et al. (2009) Abdominal obesity and the spectrum of global cardiometabolic risks in US adults. Int J Obes (Lond) 33, 239-248.

25. Cossio S, Messiah SE, Garibay-Nieto N et al. (2009) How do different indices of obesity correlate with cardiometabolic disease risk factors in multiethnic youths? Endocr Pract 15, 403-409.

26. Neufeld LM, Jones-Smith JC, Garcia R et al. (2008) Anthropometric predictors for the risk of chronic disease in non-diabetic, non-hypertensive young Mexican women. Public Health Nutr 11, 159-167.

27. Herrera VM, Casas JP, Miranda JJ et al. (2009) Interethnic differences in the accuracy of anthropometric indicators of obesity in screening for high risk of coronary heart disease. Int J Obes (Lond) 33, 568-576.

28. Perez CM, Guzman M, Ortiz AP et al. (2008) Prevalence of the metabolic syndrome in San Juan, Puerto Rico. Ethn Dis 18, 434-441.

29. Ortiz AP, Suarez E, Beauchamp G et al. (2010) Correlates of the metabolic syndrome among a sample of women in the San Juan Metropolitan Area of Puerto Rico. Metab Syndr Relat Disord 8, 235-242.

30. National Institutes of Health, National Heart, Lung, and Blood Institute (1998) Clinical Guidelines on the Identification, Evaluation, and Treatment of Overweight and Obesity in Adults: The Evidence Report. NIH Publication no. 98-4083. Washington, DC: NIH.

31. World Health Organization (2000) Obesity: Preventing and Managing the Global Epidemic. Technical Report Series no. 894. Geneva: WHO.

32. Qiao Q \& Nyamdorj R (2010) The optimal cutoff values and their performance of waist circumference and waist-to-hip ratio for diagnosing type II diabetes. Eur J Clin Nutr $\mathbf{6 4}$, 23-29.

33. Hsieh SD \& Yoshinaga H (1995) Waist/height ratio as a simple and useful predictor of coronary heart disease risk factors in women. Intern Med 34, 1147-1152.

34. Joint National Committee on Prevention, Detection, Evaluation, and Treatment of High Blood Pressure (1997) The sixth report of the Joint National Committee on Prevention, Detection, Evaluation, and Treatment of High Blood Pressure. Arch Intern Med 157, 2413-2446.

35. Expert Panel on Detection, Evaluation, and Treatment of High Blood Cholesterol in Adults (2001) Executive summary of the third report of the National Cholesterol Education Program (NCEP) Expert Panel on Detection, Evaluation, and Treatment of High Blood Cholesterol in Adults (Adult Treatment Panel III). JAMA 285, 2486-2497.

36. World Health Organization Expert Committee on Physical Status (1995) The Use of Interpretation of Anthropometry. Report of a WHO Expert Committee. Geneva: WHO.

37. American Diabetes Association (2004) Diagnosis and classification of diabetes mellitus. Diabetes Care 27, Suppl. 1, S5-S10.

38. Rexrode KM, Carey VJ, Hennekens CH et al. (1998) Abdominal adiposity and coronary heart disease in women. JAMA 280, 1843-1848.

39. Rexrode KM, Buring JE \& Manson JE (2001) Abdominal and total adiposity and risk of coronary heart disease in men. Int J Obes Relat Metab Disord 25, 1047-1056. 
40. Folsom AR, Kaye SA, Sellers TA et al. (1993) Body fat distribution and 5-year risk of death in older women. JAMA 269, 483-487.

41. de Koning L, Merchant AT, Pogue J et al. (2007) Waist circumference and waist-to-hip ratio as predictors of cardiovascular events: meta-regression analysis of prospective studies. Eur Heart J 28, 850-856.

42. Pischon T, Boeing H, Hoffmann K et al. (2008) General and abdominal adiposity and risk of death in Europe. $N$ Engl J Med 359, 2105-2120.

43. Deurenberg P, Yap M \& van Staveren WA (1998) Body mass index and percent body fat: a meta analysis among different ethnic groups. Int J Obes Relat Metab Disord 22 , 1164-1171.

44. Shen W, Punyanitya M, Chen J et al. (2006) Visceral adipose tissue: relationships between single slice areas at different locations and obesity-related health risks. Int J Obes (Lond) 31, 763-769.

45. Kotsis V, Stabouli S, Papakatsika S et al. (2010) Mechanisms of obesity-induced hypertension. Hypertens Res 33, 386-393.

46. Knowles KM, Paiva LL, Sanchez SE et al. (2011) Waist circumference, body mass index, and other measures of adiposity in predicting cardiovascular disease risk factors among Peruvian adults. Int J Hypertens 2011, 931402.

47. Lopez-Alvarenga JC, Montesinos-Cabrera RA, VelazquezAlva C et al. (2003) Short stature is related to high body fat composition despite body mass index in a Mexican population. Arch Med Res 34, 137-140.

48. Despres JP, Lemieux I, Bergeron J et al. (2008) Abdominal obesity and the metabolic syndrome: contribution to global cardiometabolic risk. Arterioscler Thromb Vasc Biol 28, 1039-1049. 